\title{
Circuit design for measure cable breakpoint location range based on AT89C52
}

\author{
Wei Youguo ${ }^{1}$, Houfei $^{1}$, Zhang Xuezhi $^{1}$, Tang Xiaokang ${ }^{1}$, Ma Yongshan $^{2}$ \\ (1.Wuhan Mechanical Technology Colledge, Wuhan, China 2.The 65370 \\ Department,Changchun,China)
}

Keyword: cable breakpoint; measure range system; AT89C52; high-frequency narrow pulse

\begin{abstract}
: the development of electric power and communication technology promotes the apply of cable widely, the trouble of cable become more and more. establish the measure cable breakpoint location range system to locate the breakpoint rapidly, the location of cable breakpoint can be calculated by the interval time that the pulse transmit and receive.

All kinds cable be used widely as the electric power and communication technology develop, the core of cable broken is a common phenomenon. And the work of examine and repair cable is more heavy. For the cable lay underground, breakpoint is covered by the protect and shield stuff, simple tools can not find breakpoint, it must adopt special way to measure the breakpoint location.So finding the breakpoint is the precondition to eliminate problem, finding the distance of breakpoint can shorten the measure time, benefit for eliminating problem and reducing loss.
\end{abstract}

\section{Status for measure cable breakpoint location range technology}

The way to locate the cable breakpoint(cable breakpoint range) can be classify two kind: calculate the breakpoint range by using the information of voltage and current, the second by using exterior signal. The first include travelling wave and impedance method, also can be classify to single port and double port way according to the resource of data; the second include test time of voice transmit and induction of voice frequency.

\section{1 test secondary pulse}

For the testing wave of sampling current is complicate, the wave of different type length 、 breakpoint range 、 impulse high voltage is various, and far from standard, it is hard to grasp the rule of wave variety. The method locate the breakpoint by using the short circuit arc when impact high voltage work on breakpoint. The method had applied in many domestic instrument, but the wave is very complicated, and can not be applied broadly.

\section{2 bridge method}

Wheatstone/Murray bridge, consist of high voltage generator 、 bridge and high sensitive galvanometer. The Wheatstone/Murray bridge is create by the proportion resistance and the core resistance between two breakpoint, when galvanometer point to zero, the bridge on balance, the resistance ratio values is equal. And according to the formula of resistance ratio, the ration of core resistance is equal to the length of cable, the breakpoint range=length* indicated ration of orientation knob. But the bridge only get the ration of breakpoint location, but can not find the exact place, still exist the defect of complexity 、 big workload and more operator.

\subsection{Impact high voltage flashover current sampling method}

The principle if method is adding a impact high voltage on the input port of trouble cable, strike out arc on breakpoint, and generate step jump voltage, and the voltage reflect between breakpoint and test port, the breakpoint range can be calculated by the time of signal travel out and home and the wave velocity of cable.

\section{4 step voltage method}

The method get the location and orientation of breakpoint by using the step voltage pulse 
descending along the soil or ground which bury the cable. according to the experience, when low voltage cable has problem, the cover of $90 \%$ breakpoint is broken. So a cycle pulse which input on one port of cable can be used to get the orientation and location along the cable. Sometimes the buried cable is not direct restricted by the terrain、 building and geography, the step voltage method still can not fulfill the location and obviation of breakpoint.

\section{Circuit design for measure cable breakpoint location range based on AT89C52}

\section{1 range measurement based on AT89C52}

The method has the advantage of online detection、 high range accuracy , good automation 、 convenient operation, solve the problem of measure error and detecting the shield cable. But arise new problem that has strict request of breakpoint range. The main reason is hardware of singlechip can not reach the demand.

For the AT89C52 has huge superiority on disposing speed, and has different mode in implement formula language, and can performance multiterm parallel sentence in one machine cycle of AT89C52, can generate more narrow pulse signal, reduce fade zone error, so it can improve the restrict of minimum range of breakpoint, and the measure accuracy is high.

\section{2 operation principle}

The operation principle of measure range system: AT89C52 single chip generate high frequency pulse for inputting to cable, the wave will reflect when meet breakpoint and end point, and reflect wave go through wave carrier、 differential amplifier、pulse reshaping circuit, receive the reflect wave by receiver. then audio frequency amplifier circuit make a sound denote receive reflect wave; and trigger single chip stop time, send a interrupt request.

Measure range system consist of CPU 、 highway , memorizer 、 transmit circuit 、 receive transmit audio alarm drive circuit and peripheral(indicator and keyboard etc), the constitute as shown in figure 2.

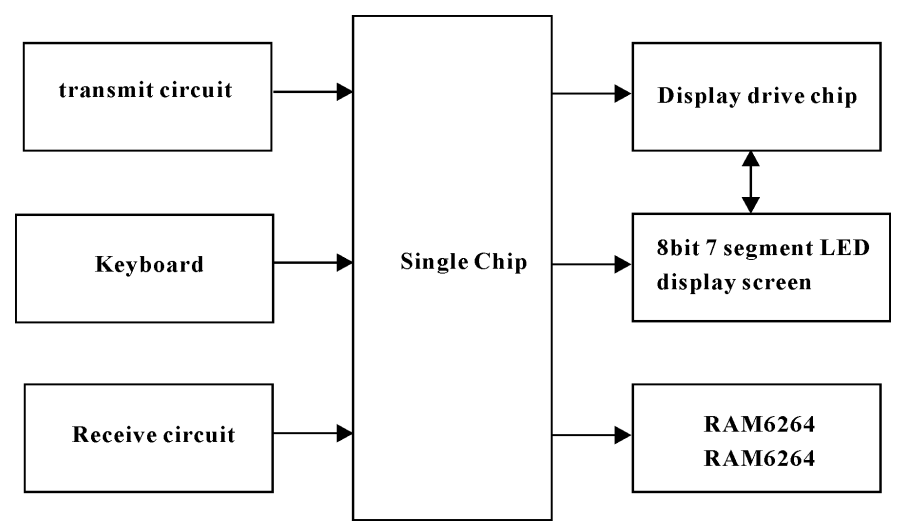

figure 1 circuit compose block

\subsection{Display circuit design}

Display circuit include flip-latch 、 encoder and driver, the circuit is complex, has much signal, so it occupy much resource of single chip, and the driver program is also complicated. The design adopt a high integration 、dynamic scan and direct drive 7 segment LED serial display interface chip MAX7219.

\subsection{Pulse transmit circuit design}

The circuit consist of photoeletric coupling circuit monostabillity trigger, phase interver high frequency emitter follower, wave carrier transmit combined circuit. Principle diagram of pulse transmit circuit as shown in figure 2. 


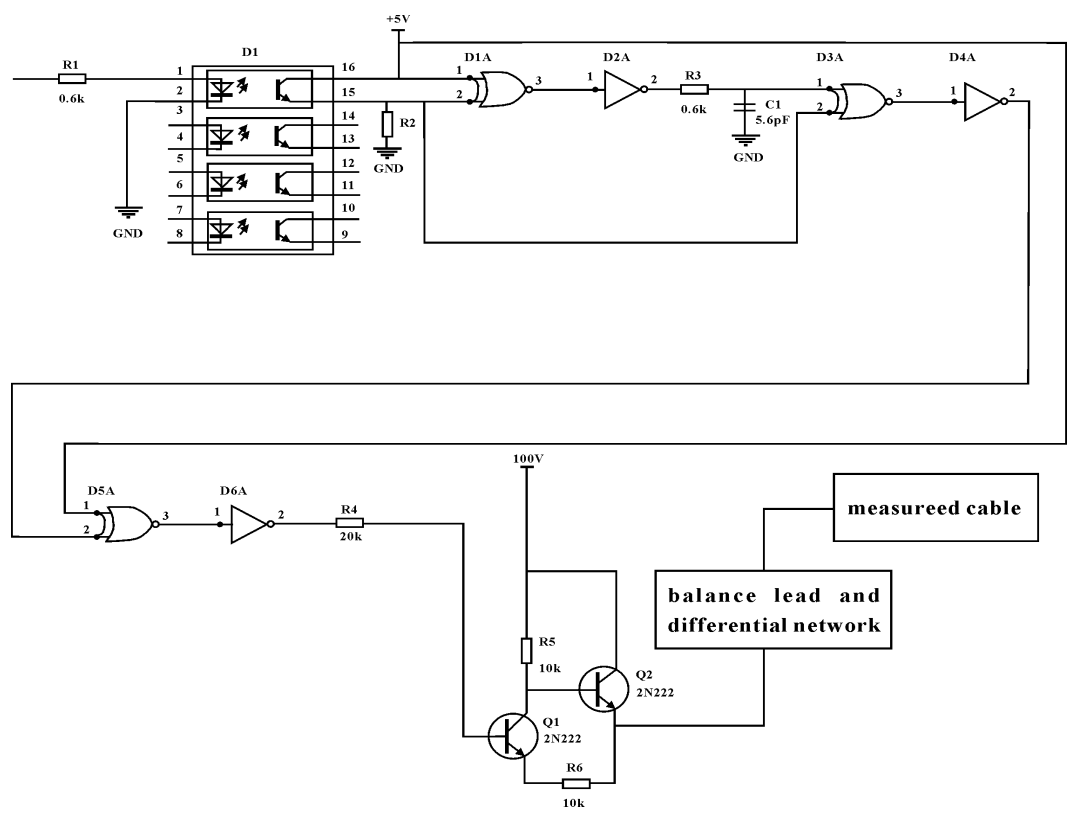

figure 2 Principle diagram of pulse transmit circuit

\subsection{Pulse receive circuit design}

Pulse receive circuit design consist of wave carrier combine、voltage decreasing amplitude、 photocoupling, pulse voltage reshaping circuit. According to the reflect character of wave, when pulse reflect to the transmit point, reflect wave shape reverse. So the reflect wave need to decreasing voltage and reshaping by two 4093 schmitt trigger. Reshaped pulse input the INT port of AT89C52 to stop time. System start to transmit operate、 display data. The principle diagram of pulse receive circuit as shown in figure 3.

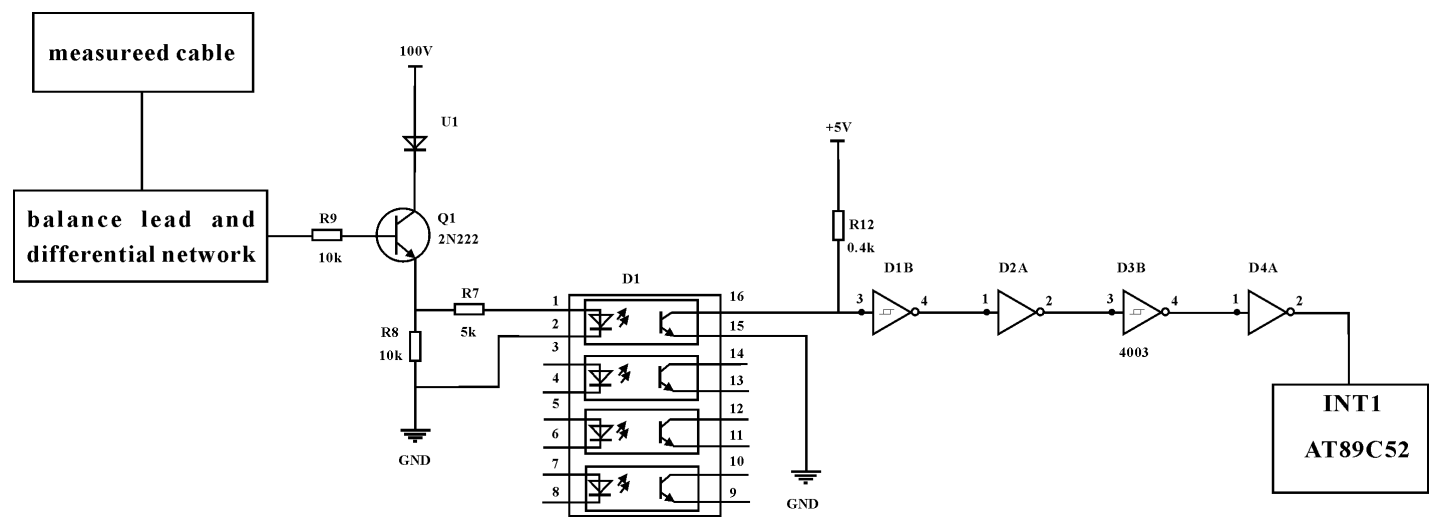

figure 3: principle diagram of pulse receive circuit

\section{Conclusion}

the system generate high-frequency, low-voltage、 narrow pulse signal, adopt photoelectric isolation by exterior transmit circuit to improve anti-jamming capacity, improve anti-load capacity by exterior radio frequency follower, then obtain the distance of breakpoint by measure the time between input port to breakpoint. so the structure of system is simple, operation is convenient, range accuracy is high, application effect is fine. 


\section{Reference:}

[1] Jiangjing Cui, Pei Liangzi, Tingxi Sun. The test and application technology of cable dectect problem. [M] high voltage technology.2001, 27(104).

[2] Boxue Tang,Huijing Miao. Principle and application of integrate circuit, electronic and industry press,2008.

[3] Ligao Li, communication cable engineering. People's posts \& telecommunication press, 2005.

[4] Cailian Zhu, Multisim Electronic circuit simulation tutorial. Xi'an Electronic and Science University press, 2007. 02, 28(1):8 - 10. 